\title{
Efficacy of echocardiography for differential diagnosis of left ventricular hypertrophy: special focus on speckle-tracking longitudinal strain
}

\author{
Hidekazu Tanaka ${ }^{1}$ iD
}

Received: 18 November 2020 / Revised: 7 December 2020 / Accepted: 13 December 2020 / Published online: 18 January 2021

(c) The Author(s) 2021

\begin{abstract}
Left ventricular (LV) hypertrophy $(\mathrm{LVH})$ is a frequent imaging finding in daily clinical practice, and its presence is associated with poor outcomes and ventricular arrhythmias. It is commonly detected in athletes, arterial hypertension, aortic stenosis, hypertrophic cardiomyopathy, cardiac amyloidosis, Fabry disease, or Friedreich's ataxia. Echocardiography plays an important role in detecting LVH and underlying causes in current clinical practice. While echocardiography is essential for the quantification and early detection of LV structural findings for various cardiovascular diseases, it has been reported that speckle-tracking echocardiographic parameters are also useful for the detection of early LV structural abnormalities. In particular, global longitudinal strain (GLS) assessed by two-dimensional speckle-tracking echocardiography is reportedly a sensitive marker for early subtle abnormalities of LV myocardial performance, helpful for the prediction of outcomes for various cardiac diseases, and superior to conventional echocardiographic indices. GLS is determined as the averaged peak longitudinal strain of $18 \mathrm{LV}$ segments from standard apical views and can be assessed as a polar plot. This polar plot longitudinal strain mapping offers an intuitive visual overview of the global and regional LV longitudinal myocardial function status of various cardiomyopathies with LVH. This mapping is clinically practicable and the plot patterns obtainable as the result of further development of this technique for clinical practice provide clues to the etiology of cardiomyopathies. This article reviews the efficacy of echocardiography for differential diagnosis of $\mathrm{LVH}$, with a special focus on the utility of speckle-tracking longitudinal strain.
\end{abstract}

Keywords Left ventricular hypertrophy $\cdot$ Speckle-tracking strain $\cdot$ Global longitudinal strain $\cdot$ Echocardiography

\section{Introduction}

Left ventricular (LV) hypertrophy (LVH) is a frequent imaging finding in daily clinical practice, and its presence is associated with poor outcomes and ventricular arrhythmias [1, 2]. It is commonly detected in athletes following long-term exercise training, in patients with arterial hypertension and aortic stenosis (AS) due to persistent pressure overload, in those with hypertrophic cardiomyopathy (HCM), as well as those with systemic diseases such as cardiac amyloidosis, Fabry disease, and Friedreich's ataxia. Echocardiography

Hidekazu Tanaka

tanakah@med.kobe-u.ac.jp

1 Division of Cardiovascular Medicine, Department of Internal Medicine, Kobe University Graduate School of Medicine,

7-5-2, Kusunoki-cho, Chuo-ku, Kobe 650-0017, Japan plays an important role in the detection of LVH and underlying causes in current clinical practice [3, 4]. While echocardiography is essential for the quantification and early detection of LV structural findings for various cardiovascular diseases [5], it has been reported that speckle-tracking echocardiographic parameters are also useful for the detection of early LV structural abnormalities. In particular, global longitudinal strain (GLS) assessed by two-dimensional speckle-tracking echocardiography is reportedly a sensitive marker for early subtle abnormalities of LV myocardial performance, helpful for the prediction of outcomes for various cardiac diseases including cardiomyopathies, and superior to conventional echocardiographic indices [6-9]. GLS is determined as the averaged peak longitudinal strain of 18 LV segments from the three standard apical views and can be assessed as a polar plot by ordinary. The polar plot longitudinal strain mapping offers an intuitive visual overview of the global and regional LV longitudinal myocardial function 
status in various cardiomyopathies with LVH. The polar plot longitudinal strain mapping is clinically practicable and the plot patterns obtainable as the result of further development of this technique in clinical practice provide clues to the etiology of cardiomyopathies, especially for patients with preserved LV ejection fraction (LVEF).

This article reviews the efficacy of echocardiography for differential diagnosis of LVH caused by arterial hypertension, HCM, cardiac amyloidosis, Fabry disease, AS, athlete's heart, and Friedreich's ataxia, with a special focus on the utility of speckle-tracking longitudinal strain. In this review article, the value of GLS is expressed as an absolute value.

\section{Arterial hypertension}

Cardiovascular diseases are common in the general population, and arterial hypertension is one of the most important risk factors for such diseases. LVH is a relatively early manifestation and a common finding in patients with arterial hypertension. As a consequence of arterial hypertension, it is associated with an increased risk of major cardiovascular events including heart failure, coronary heart disease, stroke, and sudden death $[1,10,11]$. Arterial hypertension is one of the most common causes of LVH, although the majority of patients with hypertensive LVH have a maximal interventricular septal thickness of $<15 \mathrm{~mm}$ [12].

\section{Parasternal long-axis view}

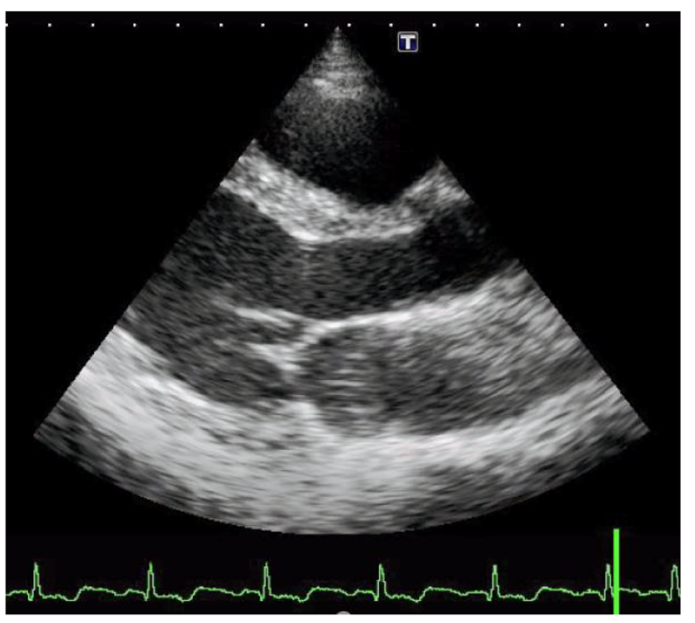

- $\mathrm{LVEF}=58 \%$

- Interventricular septum thickness $=13.0 \mathrm{~mm}$

- Posterior wall thickness $=12.0 \mathrm{~mm}$

- LV mass index $=130 \mathrm{~g} / \mathrm{m}^{2}$
GLS helps to unmask early subclinical LV systolic dysfunction in patients with arterial hypertension. The basal septum is the first segment to undergo changes under the influence of pressure overload, and GLS is further reduced at this site (Fig. 1) [13]. It was previously reported that $46 \%$ of patients showed low GLS values $(<17 \%)$, and low GLS was associated with long-lasting arterial hypertension and uncontrolled blood pressure for 200 outpatients with arterial hypertension with preserved LVEF [14]. Moreover, the degree of GLS is strongly associated with LV diastolic function, independent of changes in afterload and degree of LVH [15].

\section{Hypertrophic cardiomyopathy}

HCM is the most frequent genetically determined cardiomyopathy in adults and is characterized by non-symmetric LVH in the absence of other cardiovascular or systemic diseases. Characteristic echocardiographic findings for HCM include asymmetrical septal hypertrophy and systolic anterior motion of the mitral valve. Typically, LV end-diastolic wall thickness $\geq 15 \mathrm{~mm}$ is often observed in one or more LV myocardial segments, but isolated apical and other atypical distributions have also been reported. In cases with less wall thickening (13-14 mm), the diagnosis of $\mathrm{HCM}$ is often challenging.

It has been reported that LV longitudinal myocardial function is reduced even though LVEF is normal and may

\section{Polar plot longitudinal strain mapping}

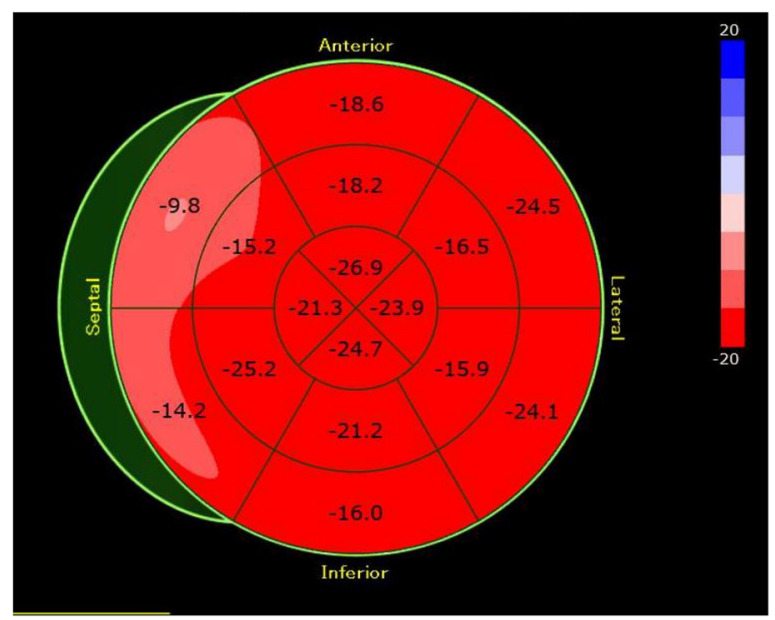

- $\mathrm{GLS}=19.8 \%$

Fig. 1 Examples of the parasternal long-axis view and the longitudinal strain bull's eye plot for a 71-year-old female with arterial hypertension. GLS of $19.8 \%$ is normal, but the longitudinal strain in the basal septum is reduced compared to that in other segments 
become abnormal before wall thickness starts to increase [16]. LV longitudinal myocardial function is typically reduced at the site of hypertrophy, especially in the region of the interventricular septum (Fig. 2) [17]. Patients with HCM were found to have similar LVEF but worse GLS compared to healthy individuals, and those with ventricular arrhythmias showed worse GLS than those without them. Therefore, GLS is an appropriate parameter for evaluating LV systolic function in HCM and may improve risk stratification of ventricular arrhythmia for such patients [18]. Moreover, abnormal GLS was found to be a better predictor of outcome for HCM than conventional echocardiographic parameters, and reduced GLS to be an independent parameter associated with poor cardiac outcomes [19].

\section{Cardiac amyloidosis}

Amyloidosis is a multi-systemic disease characterized by the deposition of amyloid fibrosis in the intercellular space of various organs [20]. Cardiac involvement occurs in up to $50 \%$ of patients with primary amyloidosis and indicates almost invariably a grave prognosis. Conventional echocardiographic features associated with cardiac amyloidosis include concentric LVH and right ventricular hypertrophy, normal LV cavity size, dilated atria, and pericardial effusion. LV diastolic abnormalities are generally recognized as the earliest manifestation of cardiac amyloidosis [21], while LV global systolic function remains normal until the later stages of the disease [22]. The myocardial texture often features a distinct "granular sparkling" appearance [23], and this echocardiographic feature is well known as a key factor in the diagnosis of cardiac amyloidosis. However, this can occur in other causes of LVH, and although high specificity rates are quoted (71-81\%), the populations studied were those referred with suspected amyloid fibrosis, so that this specificity may not be reflective of real-life practice. Moreover, sensitivity for the "granular sparkling" appearance tends to be low, with this pattern seen in only 26-36\% of cardiac amyloidosis cases [24]. It should be noted that this granular pattern applies only to standard echocardiographic imaging, without the inclusion of tissue harmonics, as this increases myocardial echogenicity in general. Newer echocardiographic image processing techniques may also reduce granular appearance. Thus, although increased echogenicity is common in cardiac amyloidosis, its usefulness as a discriminating factor is limited.

It is noteworthy that cardiac amyloidosis is characterized by regional variations in longitudinal strain from base to apex. A longitudinal strain gradient with preserved systolic strain at apical segments and significantly reduced systolic strain at mid and basal segments is consistently observed $[25,26]$. Previous studies have demonstrated that this pattern, known as "Apical Sparing", is specific, thus suited to differentiate patients with cardiac amyloidosis from patients with other causes of LVH [26, 27]. Phelan et al. compared

\section{Parasternal long-axis view}

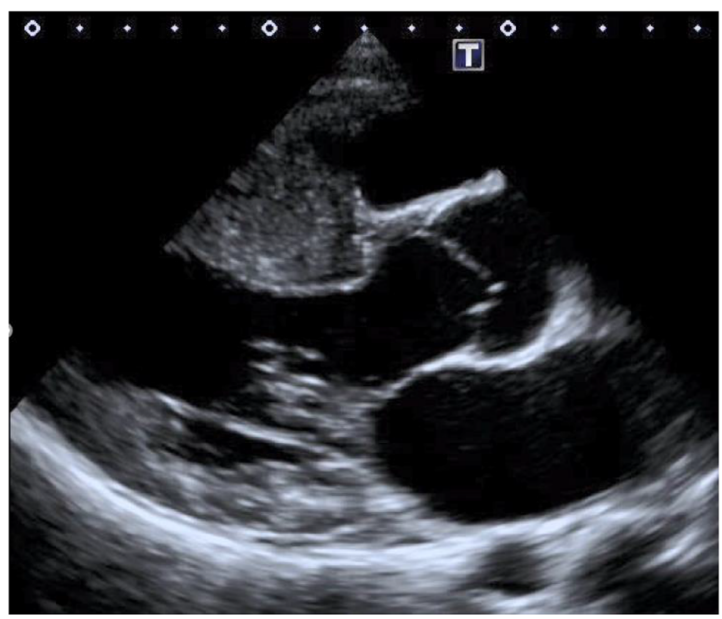

- $\mathrm{LVEF}=64 \%$

- Interventricular septum thickness $=19.2 \mathrm{~mm}$

- Posterior wall thickness $=10.2 \mathrm{~mm}$

- LV mass index $=158 \mathrm{~g} / \mathrm{m}^{2}$

\section{Polar plot longitudinal strain mapping}

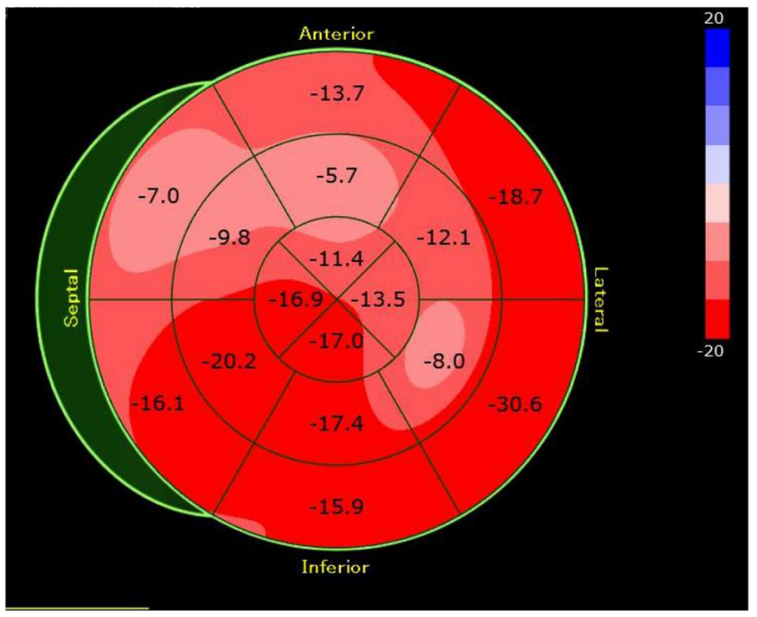

- $\mathrm{GLS}=14.7 \%$

Fig. 2 Examples of the parasternal long-axis view and the longitudinal strain bull's eye plot for a 48 -year-old male with HCM. GLS of 14.7\% is low, and longitudinal strain in the region of the interventricular septum with its increased wall thickness is further reduced 
55 consecutive patients with cardiac amyloidosis with 30 control patients with LVH comprising 15 patients with HCM and 15 with AS [25]. A relative apical longitudinal strain of 1.0 , determined by using the equation [average apical longitudinal strain/(average basal-longitudinal strain + mid-longitudinal strain)], was associated with a sensitivity of $93 \%$ and specificity of $82 \%$ for differentiating patients with cardiac amyloidosis from control patients with LVH with an area under the curve of 0.94 . Moreover, Liu et al. showed that a ratio of a septal apical-to-basal segmental longitudinal strain of $>2.1$ can differentiate cardiac amyloidosis from other causes of concentric LVH with a sensitivity of $88 \%$, specificity of $85 \%$, positive predictive value of $67 \%$, and negative predictive value of $96 \%$ [27]. This specific relative apical sparing can be easily visualized by polar plot longitudinal strain mapping for patients with cardiac amyloidosis (Fig. 3). Apical sparing is observed both in patients with transthyretin cardiac amyloidosis and in those with amyloid light-chain amyloidosis. However, the strain value at the LV apex in patients with transthyretin cardiac amyloidosis has been shown to be lower than that in patients with amyloid lightchain cardiac amyloidosis [28]. Moreover, Barros-Gomes et al. showed that GLS predicted all-cause mortality and provide additional prognostic information for all-cause mortality better than established clinical, echocardiographic, and serological markers in 150 consecutive patients with amyloid light-chain cardiac amyloidosis and preserved LVEF [29]. They also used multivariate Cox regression analysis to show that GLS was an independent predictor of all-cause mortality. In addition, the Guideline for the Diagnosis and Treatment of Cardiac Amyloidosis provided by the Japanese Circulation Society mentions that speckle-tracking echocardiography is recommended for the differentiation of cardiac amyloidosis from other causes of $\mathrm{LVH}$, and/or for the prediction of the prognosis as class I of recommendation and level $\mathrm{B}$ of evidence [30]. The speedier diagnosis of transthyretin cardiac amyloidosis by using apical sparing may therefore lead to earlier administration of tafamidis meglumine.

\section{Fabry disease}

Fabry disease is an X-linked lysosomal storage disorder caused by $\alpha$-galactosidase A deficiency. The clinical presentation is multisystemic, affecting the kidneys, heart, and nervous system. Cardiac pathophysiology is characterized by globotriaosylceramide accumulation within cardiomyocytes that cause LVH with subsequent myocardial replacement fibrosis. The most common cardiac manifestations include arrhythmias and heart failure, which are also responsible for the reduced life expectancy associated with Fabry disease [31]. Cardiomyopathy related to this disease is characterized by hypertrophy, increased LV volume, and gradual transformation to a more spherical LV shape, and myocardial replacement fibrosis. The use of the binary sign or endocardial stripe has been investigated as a hallmark of Fabry disease. The binary sign is the appearance of a bright, hyperechogenic region in the
Parasternal long-axis view

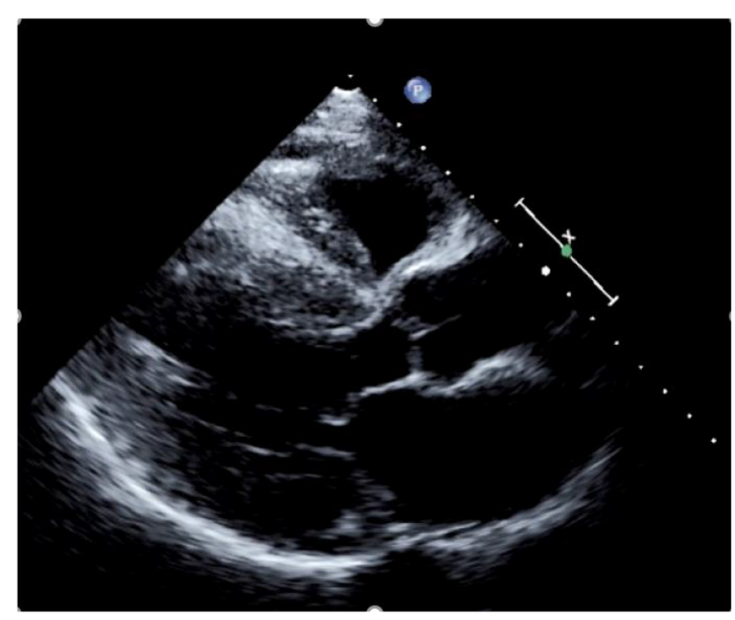

- $\mathrm{LVEF}=58 \%$

- Interventricular septum thickness $=18.9 \mathrm{~mm}$

- Posterior wall thickness $=18.2 \mathrm{~mm}$

- LV mass index $=192 \mathrm{~g} / \mathrm{m}^{2}$

\section{Polar plot longitudinal strain mapping}

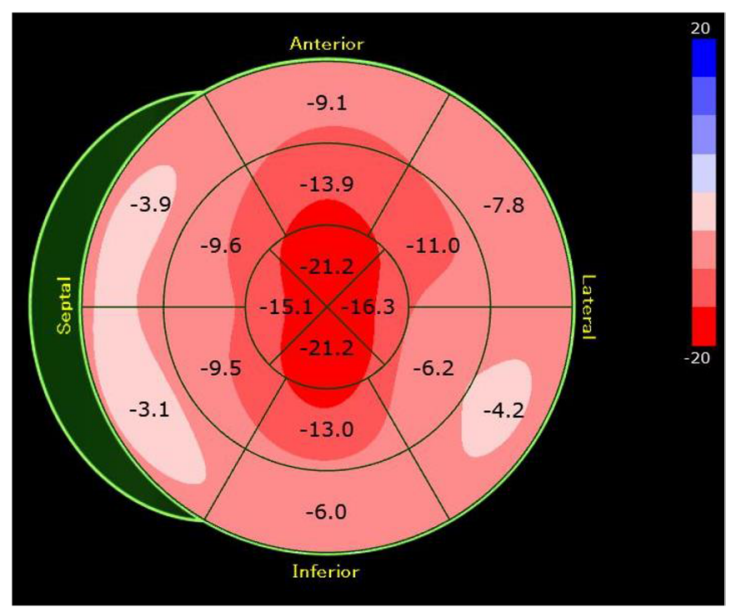

- $\mathrm{GLS}=14.2 \%$

- Relative apical longitudinal strain=1.21

- Septal apical-to-basal longitudinal strain=3.87

Fig. 3 Examples of the parasternal long-axis view and the longitudinal strain bull's eye plot for a 72-year-old male with variant transthyretin cardiac amyloidosis. GLS of $14.2 \%$ is low, while an apical sparing pattern can be observed 
LV myocardium adjacent to a relatively low echo intensity region with a clear black and white interface. Pieroni et al. found a binary sign in $83 \%$ of patients with Fabry disease [32], but other studies have demonstrated a much lower prevalence of approximately $20 \%$ and have noted that the binary sign occurs more frequently in patients with $\mathrm{LVH}$, which may partially account for this discrepancy $[33,34]$. Thus, the diagnosis of Fabry disease by means of the presence of a binary sign is of limited utility.

The development of fibrosis is often localized in the basal posterior-lateral wall even with preserved LVEF [35]. The reduced longitudinal strain in the basal lateral wall was also found at the very early stages of Fabry disease in the absence of replacement fibrosis [31]. Furthermore, Kramer et al. found no significant difference in GLS between patients with normal and elevated sphericity index among 74 Fabry disease patients with preserved LVEF [36]. In contrast, longitudinal strain in the basal posterior-lateral wall, where replacement fibrosis is usually located, is significantly decreased in patients with an elevated sphericity index. Figure 4 shows examples of the parasternal long-axis view and the longitudinal strain bull's eye plot for a 72-year-old female with Fabry disease. Her LVEF was preserved at $65 \%$ without wall motion abnormality, but GLS was as low as $12.3 \%$. Furthermore, longitudinal strain in the basal posterior-lateral wall was further reduced compared to that in other regions.

\section{Parasternal long-axis view}

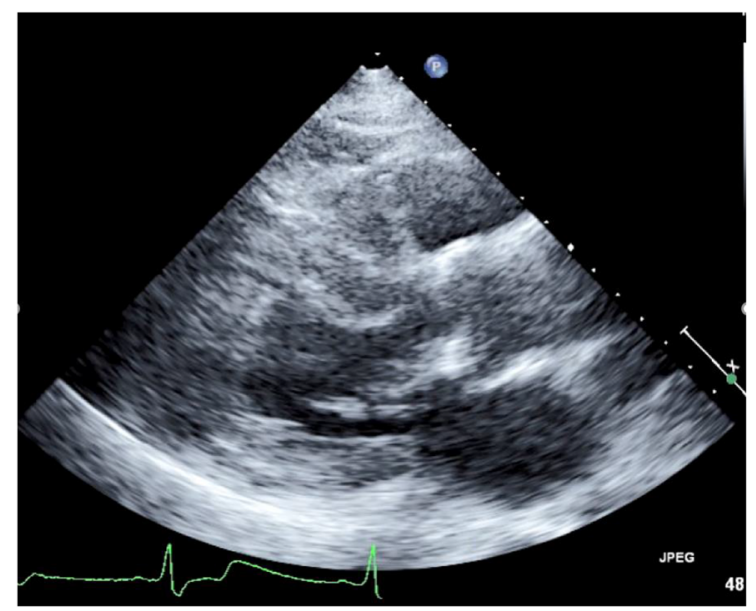

- $\mathrm{LVEF}=65 \%$

- Interventricular septum thickness $=16.8 \mathrm{~mm}$

- Posterior wall thickness $=19.2 \mathrm{~mm}$

- LV mass index $=198 \mathrm{~g} / \mathrm{m}^{2}$

Fig. 4 Examples of the parasternal long-axis view and the longitudinal strain bull's eye plot for a 72-year-old female with Fabry disease. LVEF of $65 \%$ is preserved without wall motion abnormality, but GLS

\section{Aortic stenosis}

AS is a growing health problem, and it should not be viewed as an isolated disease limited to the aortic valve, but as a systemic disease with increased peripheral vascular resistance caused by atherosclerosis and a concomitant deep alteration in LV structure, even in the presence of a preserved LVEF. LVEF is normal in most patients with AS even when symptoms develop, and valvular parameters, including transvalvular gradients and aortic valve area, are not useful for the prediction of clinical outcome after aortic valve replacement [37]. Lafitte et al. demonstrated that patients with severe AS and preserved LVEF had lower GLS compared with matched control subjects, and that this difference was more pronounced in the basal LV segments. A lower GLS was also found to be associated with a higher LV mass index and relative wall thickness, which supports the notion of a direct connection between concentric remodeling and contractile dysfunction [38]. From a physiological point of view, the pressure overload of AS triggers a continuum of changes that start from myocyte hypertrophy and interstitial reactive fibrosis, leading to a self-perpetuating process of cellular atrophy, myocyte death, and replacement fibrosis, eventually causing a progressive deterioration of myocardial function with poor prognosis [39].

AS combined with transthyretin cardiac amyloidosis has attracted attention in recent years, because their

\section{Polar plot longitudinal strain mapping}

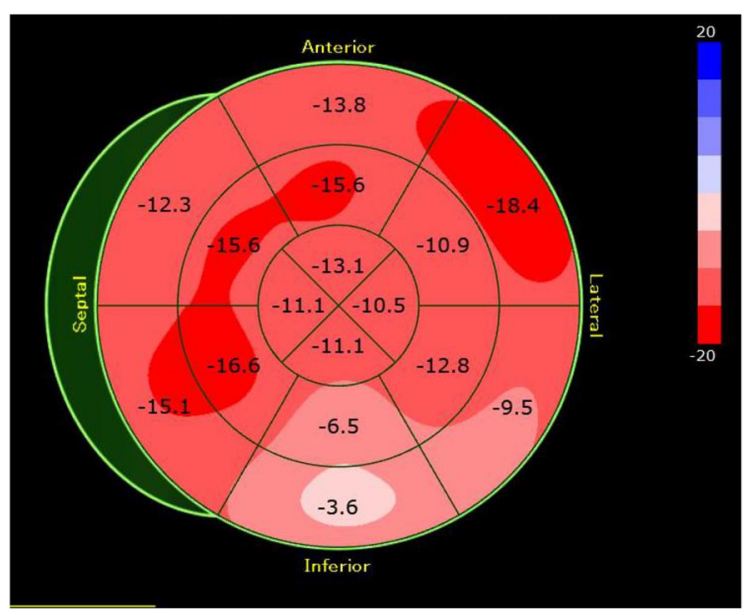

- $\mathrm{GLS}=12.3 \%$

of $12.3 \%$ is low. Furthermore, longitudinal strain in the basal posterior-lateral wall was further reduced compared to that in other regions 
coexistence must always be taken into consideration since both transthyretin cardiac amyloidosis and severe AS result in $\mathrm{LVH}$, thus interfering with the detection of suspected coexistence of transthyretin cardiac amyloidosis. Treibel et al. reported that the prevalence of wild-type transthyretin cardiac amyloid was $6 \%$ among patients with severe AS aged $>65$ years undergoing surgical aortic valve replacement, and was associated with a poor outcome [40]. In addition, Castaño et al. reported that transthyretin cardiac amyloidosis was prevalent in $16 \%$ of patients with severe AS undergoing transcatheter aortic valve implantation and was associated with a severe low-flow low-gradient AS phenotype despite only mildly reduced LVEF [41]. Another study observed occult cardiac amyloidosis in $13.9 \%$ of severe aortic stenosis patients prior to transcatheter aortic valve implantation [42]. Thus, the presence of transthyretin cardiac amyloidosis can be suspected based on various red flags, especially apical sparing, in severe AS patients who have been referred for transcatheter aortic valve implantation or surgical aortic valve replacement. This detection procedure has become especially significant since tafamidis meglumine was launched for the treatment of transthyretin cardiac amyloidosis (Fig. 5).

Parasternal long-axis view

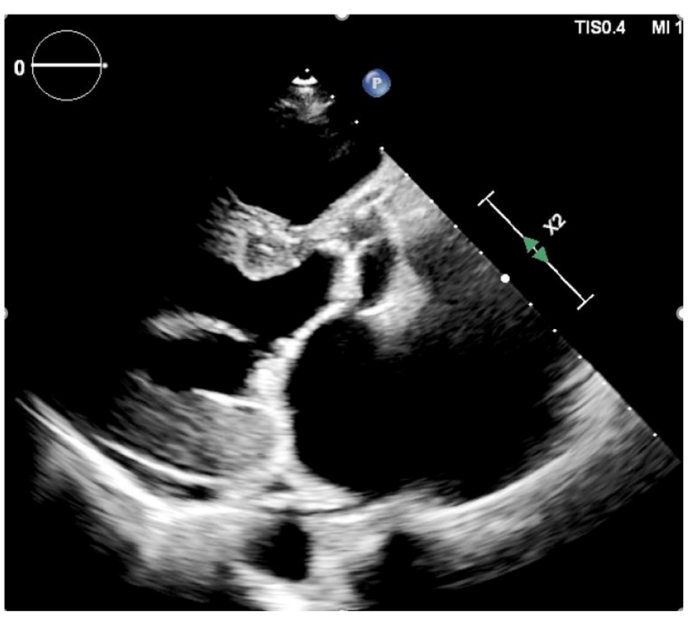

- $\mathrm{LVEF}=57 \%$

- Interventricular septum thickness $=12.9 \mathrm{~mm}$

- Posterior wall thickness $=16.0 \mathrm{~mm}$

- LV mass index $=188 \mathrm{~g} / \mathrm{m}^{2}$

- Peak aortic jet velocity $=4.4 \mathrm{~m} / \mathrm{s}$

- Mean aortic pressure gradient $=42 \mathrm{mmHg}$

- Aortic valve area $=0.70 \mathrm{~cm}^{2}$

Fig. 5 Examples of the parasternal long-axis view and the longitudinal strain bull's eye plot for an 82-year-old male with severe AS who was referred for transcatheter aortic valve implantation. GLS of $9.8 \%$

\section{Athlete's heart}

Physiological hypertrophy can be detected in the heart of athletes, whose LV dilation and LVH may be pronounced enough to mimic a pathological state, but LV systolic and diastolic function is normal or even supranormal. In toplevel athletes, LV end-diastolic diameter is often increased, although LVEF is normal, with normal or supranormal stroke volume and systolic peak velocity $>9 \mathrm{~cm} / \mathrm{s}$ by means of tissue Doppler imaging [43]. Some athletes may present enhanced early diastolic LV filling [44], so that assessment of LV diastolic function might be a key factor for differentiating physiological $\mathrm{LVH}$ due to adaptation to exercise from pathological LVH [45]. Identification of cardiomyopathies can be challenging when the wall thickness is between 12 and $16 \mathrm{~mm}$ (the so-called gray zone of LVH). Kansal et al. reported the utility of conventional echocardiographic and speckle-tracking parameters for distinguishing the pathologic LVH of hypertrophic cardiomyopathy from the physiologic LVH of professional football players when septal wall thickness falls within a "gray zone" between 12 and $16 \mathrm{~mm}$ [46]. They studied 28 professional football players and 21 patients with HCM, with septal wall thicknesses of 12-16 mm, along with 17 normal controls. They showed

\section{Polar plot longitudinal strain mapping}

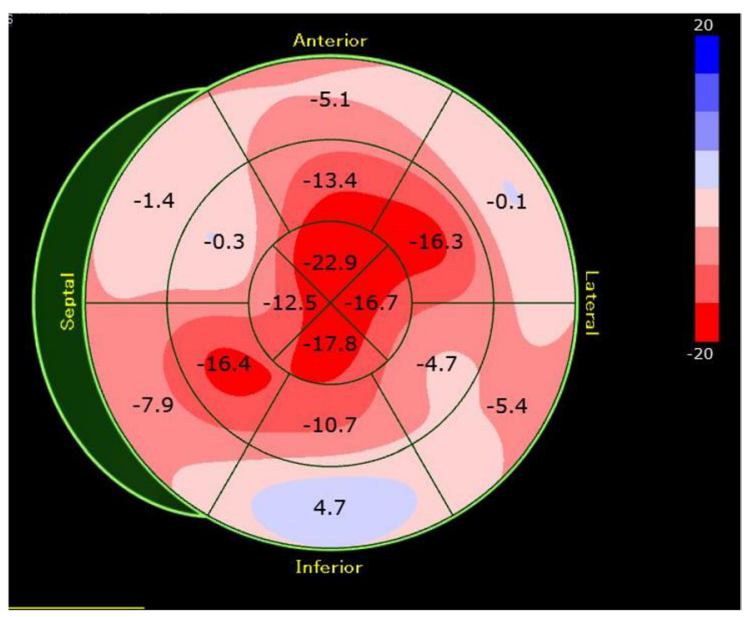

- $\mathrm{GLS}=9.8 \%$

- Relative apical longitudinal strain=1.21

- Septal apical-to-basal longitudinal strain=8.93

is low, but an apical sparing pattern can be observed. Moreover, the diagnosis of this patient was complicated by wild-type transthyretin cardiac amyloidosis 
Table 1 Strain pattern in different models of LVH

\begin{tabular}{lll}
\hline Type of LVH & GLS value & Typical impairment of longitudinal strain \\
\hline $\begin{array}{l}\text { Arterial hypertension } \\
\text { Hypertrophic cardiomyopathy }\end{array}$ & $\begin{array}{l}\text { Normal or reduced } \\
\text { Reduced }\end{array}$ & $\begin{array}{l}\text { Basal septum } \\
\text { The site of hypertrophy, especially in the } \\
\text { region of the interventricular septum }\end{array}$ \\
Cardiac amyloidosis & Reduced & $\begin{array}{c}\text { Regional variations in longitudinal strain } \\
\text { from base-to-apex (Apical sparing) }\end{array}$ \\
Fabry disease & Reduced & Basal posterior-lateral wall \\
Aortic stenosis & Reduced & Basal LV segments \\
Athlete's heart & Almost normal & None \\
Friedreich's ataxia & Mildly reduced & None \\
\hline
\end{tabular}

that relative wall thickness and GLS of patients with HCM were significantly larger than those of professional football players. They also showed that relative wall thickness most accurately differentiated professional football players from patients with HCM. Afonso et al. also compared GLS of 56 patients with HCM, 34 patients with LVH due to arterial hypertension, 27 professional athletes with LVH and 12 healthy controls in sinus rhythm with preserved LVEF [47]. They showed that GLS of professional athletes with LVH $(17.8 \pm 2.2 \%)$ was mildly reduced compared to healthy controls $(18.7 \pm 1.8 \%)$, and was similar to that of patients with LVH due to arterial hypertension $(17.8 \pm 3.1 \%)$, but significantly higher compared to that of patients with HCM $(11.2 \pm 4.2 \%)$.

\section{Friedreich's ataxia}

Friedreich's ataxia is an autosomal recessive neurodegenerative disease caused by a guanine-adenine-adenine triplet repeat expansion in the first intron of frataxin [50]. The intronic expansion leads to a specific iron-sulfur protein deficiency, resulting in intra-mitochondrial iron accumulation. Besides the neurologic manifestation, cardiac involvement and endocrine involvement are also frequently observed [27]. A concentric LVH with an end-diastolic wall thickness of less than $15 \mathrm{~mm}$ is the usual echocardiographic feature [48]. Around $40 \%$ of patients with Friedreich's ataxia show concentric remodeling, 35\% concentric hypertrophy, and only 5\% eccentric hypertrophy [49]. Global LV systolic and diastolic function remain normal in most patients with Friedreich's ataxia, and only end-stage patients with this disease develop reduced LVEF with global hypokinesia and slightly dilated LV chamber[3]. Electrocardiographic abnormalities are often the earliest sign of Friedreich's ataxia. At this early stage, echocardiography results are usually normal and the polar plot longitudinal strain mapping shows a similar pattern as that of healthy subjects. In patients with Friedreich's ataxia with concentric LVH and normal LVEF, the polar plot longitudinal strain mapping pattern indicates a mildly reduced GLS [50]. Myocardial fibrosis develops gradually, leading to LV wall thinning and LV dilatation during the disease progression, while LVEF remains preserved for a long time until the end-stage of the disease [48]. Noteworthy is that the LV wall thinning appears to be diffuse in patients with Friedreich's ataxia, which is different from the typical findings for Fabry cardiomyopathy. The polar plot longitudinal strain mapping shows significantly reduced GLS when LVEF is reduced. Additionally, Friedreich's ataxia shares some echocardiographic features with cardiac amyloidosis in terms of morphology, including concentric LVH with a sparkling granular texture of myocardium. Different from cardiac amyloidosis, though, LV diastolic function of patients with Friedreich's ataxia can be normal or only mildly impaired. Moreover, a longitudinal base-to-apex strain gradient, which is frequently evidenced in cardiac amyloidosis, is rarely detected in patients with Friedreich's ataxia [27]. Furthermore, the underlying mechanisms of myocardial dysfunction in patients with Friedreich's ataxia might be associated with myocyte cellular hypertrophy, iron deposits, focal necrosis, and diffuse fibrosis [51].

\section{Conclusion}

Detection of a disease-related typical deformation pattern and polar plot longitudinal strain mapping may provide valuable clues for the final diagnosis in some patients with unclear LVH (Table 1).

\section{Compliance with ethical standards}

Conflict of interest Hidekazu Tanaka has received a speaker honorarium from Boehringer Ingelheim $\mathrm{GmbH}$, Sumitomo Dainippon Pharma, AstraZeneca PLC, Mitsubishi Tanabe Pharma Corporation, Taisho Pharma Co., Ltd., TOA EIYO LTD, Bayer Yakuhin, Ltd, ONO PHARMACEUTICAL CO., LTD., General Electric Company, Daiichi Sankyo Company, Limited, Novartis International AG, Teijin Limited, Merck \& Co., Inc., Sekisui Medical Company, Limited, and Philips Medical Systems. 
Open Access This article is licensed under a Creative Commons Attribution 4.0 International License, which permits use, sharing, adaptation, distribution and reproduction in any medium or format, as long as you give appropriate credit to the original author(s) and the source, provide a link to the Creative Commons licence, and indicate if changes were made. The images or other third party material in this article are included in the article's Creative Commons licence, unless indicated otherwise in a credit line to the material. If material is not included in the article's Creative Commons licence and your intended use is not permitted by statutory regulation or exceeds the permitted use, you will need to obtain permission directly from the copyright holder. To view a copy of this licence, visit http://creativecommons.org/licenses/by/4.0/.

\section{References}

1. Verdecchia P, Carini G, Circo A, et al. Left ventricular mass and cardiovascular morbidity in essential hypertension: the MAVI study. J Am Coll Cardiol. 2001;38:1829-35.

2. Draper TS Jr, Silver JS. Gaasch WH adverse structural remodeling of the left ventricle and ventricular arrhythmias in patients with depressed ejection fraction. J Card Fail. 2015;21:97-102.

3. Weidemann F, Niemann M, Ertl G, et al. The different faces of echocardiographic left ventricular hypertrophy: clues to the etiology. J Am Soc Echocardiogr. 2010;23:793-801.

4. Cikes M, Sutherland GR, Anderson LJ, et al. The role of echocardiographic deformation imaging in hypertrophic myopathies. Nat Rev Cardiol. 2010;7:384-96.

5. Ponikowski P, Voors AA, Anker SD, et al. 2016 ESC Guidelines for the diagnosis and treatment of acute and chronic heart failure: the Task Force for the diagnosis and treatment of acute and chronic heart failure of the European Society of Cardiology (ESC) Developed with the special contribution of the Heart Failure Association (HFA) of the ESC. Eur Heart J. 2016;37:2129-200.

6. Tanaka H. Utility of strain imaging in conjunction with heart failure stage classification for heart failure patient management. J Echocardiogr. 2019;17:17-24.

7. Gorcsan J 3rd, Tanaka H. Echocardiographic assessment of myocardial strain. J Am Coll Cardiol. 2011;58:1401-13.

8. Biering-Sorensen T, Biering-Sorensen SR, Olsen FJ, et al. Global longitudinal strain by echocardiography predicts long-term risk of cardiovascular morbidity and mortality in a low-risk general population: the Copenhagen City Heart Study. Circ Cardiovasc Imaging. 2017;10.

9. Stanton T, Leano R. Marwick TH prediction of all-cause mortality from global longitudinal speckle strain: comparison with ejection fraction and wall motion scoring. Circ Cardiovasc Imaging. 2009;2:356-64.

10. Koren MJ, Devereux RB, Casale PN, et al. Relation of left ventricular mass and geometry to morbidity and mortality in uncomplicated essential hypertension. Ann Intern Med. 1991;114:345-52.

11. Casale PN, Devereux RB, Milner M, et al. Value of echocardiographic measurement of left ventricular mass in predicting cardiovascular morbid events in hypertensive men. Ann Intern Med. 1986;105:173-8.

12. Elliott TF, Anastasakis A, Authors/Task Force m, et al. ESC Guidelines on diagnosis and management of hypertrophic cardiomyopathy: the Task Force for the Diagnosis and Management of Hypertrophic Cardiomyopathy of the European Society of Cardiology (ESC). Eur Heart J. 2014;2014(35):2733-79.

13. Galderisi M, Lomoriello VS, Santoro A, et al. Differences of myocardial systolic deformation and correlates of diastolic function in competitive rowers and young hypertensives: a speckletracking echocardiography study. J Am Soc Echocardiogr. 2010;23:1190-8.
14. Bendiab STN, Meziane-Tani A, Ouabdesselam S, et al. Factors associated with global longitudinal strain decline in hypertensive patients with normal left ventricular ejection fraction. Eur J Prev Cardiol. 2017;24:1463-72.

15. Galderisi M, Esposito R, Schiano-Lomoriello V, et al. Correlates of global area strain in native hypertensive patients: a threedimensional speckle-tracking echocardiography study. Eur Heart J Cardiovasc Imaging. 2012;13:730-8.

16. Losi MA, Nistri S, Galderisi M, et al. Echocardiography in patients with hypertrophic cardiomyopathy: usefulness of old and new techniques in the diagnosis and pathophysiological assessment. Cardiovasc Ultrasound. 2010;8:7.

17. Urbano-Moral JA, Rowin EJ, Maron MS, et al. Investigation of global and regional myocardial mechanics with 3-dimensional speckle tracking echocardiography and relations to hypertrophy and fibrosis in hypertrophic cardiomyopathy. Circ Cardiovasc Imaging. 2014;7:11-9.

18. Haland TF, Almaas VM, Hasselberg NE, et al. Strain echocardiography is related to fibrosis and ventricular arrhythmias in hypertrophic cardiomyopathy. Eur Heart J Cardiovasc Imaging. 2016;17:613-21.

19. Reant P, Mirabel M, Lloyd G, et al. Global longitudinal strain is associated with heart failure outcomes in hypertrophic cardiomyopathy. Heart. 2016;102:741-7.

20. Merlini G, Bellotti V. Molecular mechanisms of amyloidosis. N Engl J Med. 2003;349:583-96.

21. Klein AL, Hatle LK, Taliercio CP, et al. Serial Doppler echocardiographic follow-up of left ventricular diastolic function in cardiac amyloidosis. J Am Coll Cardiol. 1990;16:1135-41.

22. Swanton RH, Brooksby IA, Davies MJ, et al. Systolic and diastolic ventricular function in cardiac amyloidosis. Studies in six cases diagnosed with endomyocardial biopsy. Am J Cardiol. 1977;39:658-64.

23. Siqueira-Filho AG, Cunha CL, Tajik AJ, et al. M-mode and twodimensional echocardiographic features in cardiac amyloidosis. Circulation. 1981;63:188-96.

24. Selvanayagam JB, Hawkins PN, Paul B, et al. Evaluation and management of the cardiac amyloidosis. J Am Coll Cardiol. 2007;50:2101-10.

25. Phelan D, Collier P, Thavendiranathan P, et al. Relative apical sparing of longitudinal strain using two-dimensional speckletracking echocardiography is both sensitive and specific for the diagnosis of cardiac amyloidosis. Heart. 2012;98:1442-8.

26. Liu D, Hu K, Niemann M, et al. Impact of regional left ventricular function on outcome for patients with AL amyloidosis. PLoS ONE. 2013;8:e56923.

27. Liu D, Hu K, Niemann M, et al. Effect of combined systolic and diastolic functional parameter assessment for differentiation of cardiac amyloidosis from other causes of concentric left ventricular hypertrophy. Circ Cardiovasc Imaging. 2013;6:1066-72.

28. Tuzovic M, Yang EH, Baas AS, et al. Cardiac amyloidosis: diagnosis and treatment strategies. Curr Oncol Rep. 2017;19:46.

29. Barros-Gomes S, Williams B, Nhola LF, et al. Prognosis of light chain amyloidosis with preserved LVEF: added value of 2D speckle-tracking echocardiography to the current prognostic staging system. JACC Cardiovasc Imaging. 2017;10:398-407.

30. Kitaoka H, Izumi C, Izumiya Y, et al. JCS 2020 Guideline on Diagnosis and Treatment of Cardiac Amyloidosis. Circ J. 2020;84:1610-71.

31. Weidemann F, Breunig F, Beer M, et al. The variation of morphological and functional cardiac manifestation in Fabry disease: potential implications for the time course of the disease. Eur Heart J. 2005;26:1221-7.

32. Pieroni M, Chimenti C, De Cobelli F, et al. Fabry's disease cardiomyopathy: echocardiographic detection of endomyocardial 
glycosphingolipid compartmentalization. J Am Coll Cardiol. 2006;47:1663-71.

33. Kounas S, Demetrescu C, Pantazis AA, et al. The binary endocardial appearance is a poor discriminator of Anderson-Fabry disease from familial hypertrophic cardiomyopathy. J Am Coll Cardiol. 2008;51:2058-61.

34. Mundigler G, Gaggl M, Heinze G, et al. The endocardial binary appearance ('binary sign') is an unreliable marker for echocardiographic detection of Fabry disease in patients with left ventricular hypertrophy. Eur J Echocardiogr. 2011;12:744-9.

35. Kramer J, Niemann M, Liu D, et al. Two-dimensional speckle tracking as a non-invasive tool for identification of myocardial fibrosis in Fabry disease. Eur Heart J. 2013;34:1587-96.

36. Kramer J, Bijnens B, Stork S, et al. Left ventricular geometry and blood pressure as predictors of adverse progression of Fabry cardiomyopathy. PLoS ONE. 2015;10:e0140627.

37. Hachicha Z, Dumesnil JG, Bogaty P, et al. Paradoxical low-flow, low-gradient severe aortic stenosis despite preserved ejection fraction is associated with higher afterload and reduced survival. Circulation. 2007;115:2856-64.

38. Lafitte $\mathrm{S}$, Perlant $\mathrm{M}$, Reant $\mathrm{P}$, et al. Impact of impaired myocardial deformations on exercise tolerance and prognosis in patients with asymptomatic aortic stenosis. Eur J Echocardiogr. 2009;10:414-9.

39. Hein S, Arnon E, Kostin S, et al. Progression from compensated hypertrophy to failure in the pressure-overloaded human heart: structural deterioration and compensatory mechanisms. Circulation. 2003;107:984-91.

40. Treibel TA, Fontana M, Gilbertson JA, et al. Occult transthyretin cardiac amyloid in severe calcific aortic stenosis: prevalence and prognosis in patients undergoing surgical aortic valve replacement. Circ Cardiovasc Imaging. 2016;9:e005066.

41. Castano A, Narotsky DL, Hamid N, et al. Unveiling transthyretin cardiac amyloidosis and its predictors among elderly patients with severe aortic stenosis undergoing transcatheter aortic valve replacement. Eur Heart J. 2017;38:2879-87.

42. Scully PR, Treibel TA, Fontana M, et al. Prevalence of cardiac amyloidosis in patients referred for transcatheter aortic valve replacement. J Am Coll Cardiol. 2018;71:463-4.

43. Pelliccia A, Maron BJ, Spataro A, et al. The upper limit of physiologic cardiac hypertrophy in highly trained elite athletes. N Engl J Med. 1991;324:295-301.
44. D'Andrea A, Cocchia R, Riegler L, et al. Left ventricular myocardial velocities and deformation indexes in top-level athletes. J Am Soc Echocardiogr. 2010;23:1281-8.

45. Caso P, D’Andrea A, Galderisi M, et al. Pulsed Doppler tissue imaging in endurance athletes: relation between left ventricular preload and myocardial regional diastolic function. Am J Cardiol. 2000;85:1131-6.

46. Kansal MM, Lester SJ, Surapaneni P, et al. Usefulness of twodimensional and speckle tracking echocardiography in "Gray Zone" left ventricular hypertrophy to differentiate professional football player's heart from hypertrophic cardiomyopathy. Am J Cardiol. 2011;108:1322-6.

47. Afonso L, Kondur A, Simegn M, et al. Two-dimensional strain profiles in patients with physiological and pathological hypertrophy and preserved left ventricular systolic function: a comparative analyses. BMJ Open. 2012;17(2):e001390.

48. Weidemann F, Rummey C, Bijnens B, et al. The heart in Friedreich ataxia: definition of cardiomyopathy, disease severity, and correlation with neurological symptoms. Circulation. 2012;125:1626-34.

49. Regner SR, Lagedrost SJ, Plappert T, et al. Analysis of echocardiograms in a large heterogeneous cohort of patients with Friedreich ataxia. Am J Cardiol. 2012;109:401-5.

50. Sutton SJM, Ky B, Regner SR, et al. Longitudinal strain in Friedreich ataxia: a potential marker for early left ventricular dysfunction. Echocardiography. 2014;31:50-7.

51. Puccio H, Simon D, Cossee M, et al. Mouse models for Friedreich ataxia exhibit cardiomyopathy, sensory nerve defect and Fe-S enzyme deficiency followed by intramitochondrial iron deposits. Nat Genet. 2001;27:181-6.

Publisher's Note Springer Nature remains neutral with regard to jurisdictional claims in published maps and institutional affiliations. 